\title{
Cobalt doped JUC-160 derived functional carbon superstructures with synergetic catalyst effect for $\mathrm{Li}-\mathrm{SeS}_{2}$ batteries
}

\author{
Wen-wu Jin ${ }^{a}$, He-Jun Li ${ }^{b}$, Ji-zhao Zou ${ }^{a}$, Qi Zhang ${ }^{c}$, Saikumar Inguvad, Shao-zhong \\ Zeng $^{\mathrm{a}}$, Guo-zhong $\mathrm{Xu}^{\mathrm{a}}$, Xie-rong Zeng ${ }^{\mathrm{a}}$
}

\begin{abstract}
${ }^{a}$ Shenzhen Key Laboratory of Special Functional Materials \& Shenzhen Engineering Laboratory for Advance Technology of Ceramics, College of Materials Science and Engineering, Shenzhen University, Shenzhen, 518060, PR China
\end{abstract}

b State Key Laboratory of Solidification Processing, Carbon/Carbon Composites Research Center, Northwestern
Polytechnical University, Xi'an, 710072, PR China

${ }^{\mathrm{c}}$ School of Aerospace, Transport and Manufacturing, Cranfield University, Cranfield, Bedfordshire, MK43 OAL, UK

${ }^{\mathrm{d}}$ School of Chemistry, University College Cork and Tyndall National Institute, Cork, T12YN60, Ireland

\section{Introduction}

Mobile energy storage capacity is considered as the core to measure the cruising ability of an electric vehicle. Therefore, the research and development of power battery has attracted a great attention in recent years. Li-S batteries with a high theoretical energy density are considered as excellent candidates to replace commercial lithium batteries [1]. Unfortunately, sulfur insulation leads to the low utilization rate of the active substances and poor rate performance of the Li-S batteries. Selenium (Se) is in the same group as sulfur, it has higher electronic conductivity, and was proposed as a cathode material [2]. However, Se is very expensive, which greatly limits the large-scale commercial production of Li-Se batteries. In order to make a full use of the high conductivity of selenium $\left(10^{-3} \mathrm{~S} / \mathrm{cm}\right)$ and the high theoretical energy density of sulfur $\left(2600 \mathrm{Wh} \cdot \mathrm{kg}^{-1}\right), \mathrm{SeS}_{2}$ were first proposed as electrode materials by Abouimrane et al. $[3,4]$ Previous studies have proven that the $\mathrm{SeS}_{2}$ electrode is suitable for the battery systems of lithium, sodium and potassium-ion [[5], [6], [7], [8]]. Therefore, the influence of the metal price on the commercial production has been freed. However, Li$\mathrm{SeS}_{2}$ battery is still affected by shuttle effect, which will reduce the utilization of active substances and $\mathrm{Li}$ anode corrosion, therefore, the electrochemical performance of $\mathrm{Li}$ $\mathrm{SeS}_{2}$ batteries are greatly limited [9]. Utilizing the carbon/ $\mathrm{SeS}_{2}$ electrode co-doped with metal/heteroatom is considered as a cost-effective method to address these challenges. These carbon/SeS $\mathrm{S}_{2}$ cathode materials can anchor polysulfide/selenium compounds through chemical constraints, thus significantly improving the electrochemical performance of the Li$\mathrm{SeS}_{2}$ batteries. Therefore, the carbonaceous materials with polar metal [10], metal nitride $[11,12]$ and metal sulfides $[9,13]$ have been widely used in the field of $\mathrm{Li}^{-} \mathrm{SeS}_{2}$ batteries.

Zeolites based imidazoliates framework (ZIF) is a kind of zeolite-like metal-organic framework (MOF). More than 150 ZIF structures have been identified since the first ZIF was reported in 2006 [[14], [15], [16], [17]]. Due to its various crystal structure and high thermal stability, the ZIF is considered as an ideal candidate for preparing functional carbon materials [16]. Unfortunately, the direct carbonization products of MOF usually result in the microporous structure [18], which greatly limits the loading capacity of the MOF-derived carbon matrix to active substances. In order to prepare carbon electrode materials with hierarchical porous structure, it is usually necessary to optimize the structure of MOF. With the development of crystal-shape engineering of the MOF in recent years, it is found that the framework structure 
of MOF can be effectively controlled by adjusting the organic ligands or central ions [17,[19], [20], [21], [22], [23], [24], [25]]. The recent discoveries provide a theoretical basis for tailoring the structure of the MOF. For example, Fischer's group has used the method of de novo synthesis and post-synthetic treatment to make targeted modifications of the organic ligand. Because of this process benefits, a large number of defects can be introduced into MOF crystals [19,20]. Similarly, the framework structure of the MOF can also be tailored by modifying the metal ions, such as $\mathrm{Zn}^{2+}, \mathrm{Co}^{2+}$ and $\mathrm{Fe}^{2+}$ [[21], [22], [23], [24], [25]]. Based on the above advantages, MOF materials have been widely used in the field of $\mathrm{Li}-\mathrm{Se}$ and $\mathrm{Li}-\mathrm{S}$ batteries $[2,26]$. However, to the best of our knowledge, MOF derived carbon materials are rarely reported in the field of $\mathrm{Li}_{-} \mathrm{SeS}_{2}$ batteries [10,12,27]. Moreover, the application of MOF derived self-assembled cathode materials has not been reported in this area.

Inspired by the above considerations, the crystal-shape engineering of Zn-based ZIF (JUC-160) was carried out. For the first time, the self-assembled carbon nanostructures derived from ZIF were employed as carbon matrixes to embed $\mathrm{SeS}_{2}$ for $\mathrm{Li}-\mathrm{SeS} \mathrm{S}_{2}$ batteries, and the physicalchemical properties of the carbon nanostructures can be further tailored by adjusting the amount of cobalt source and the cobalt doping method. This treatment opens a new road to prepare cobalt/nitrogen co-doped carbon materials with self-assembled nanostructures, which are favorable for charge-transfer and anchoring the polysulfides/polyselenides through strong chemical interactions. Therefore, an excellent electrochemical performance was observed for the optimized electrode. Even if the current is increased to $10 \mathrm{C}$, the reversible specific capacity of CNC-0.03/SeS $2, \mathrm{CNC}-0.05 / \mathrm{SeS}_{2}$ and CNC-0.07/SeS 2 can still be maintained at 203.9, 158.9 and $234.0 \mathrm{~mA} \mathrm{~h} \mathrm{~g}^{-1}$, respectively, exhibiting excellent rate performances. In addition, the CNC-0.05/SeS 2 displays extraordinary cycle performance. Even after 250 cycles at $0.5 \mathrm{C}$, the $\mathrm{CNC}-0.05 / \mathrm{SeS}_{2}$ still displays a reversible capacity of $760.93 \mathrm{~mA} \mathrm{~h} \mathrm{~g}^{-1}$ with reversible chargedischarge efficiency close to $100 \%$.

\section{Experimental details}

\subsection{Synthesis of JUC-160}

Typically [28], benzimidazole (1.1814g) and 2-methylbenzimidazole (1.05728 g) were dissolved in $160 \mathrm{ml}$ DMF (N,N-Dimethylformamide) to form a clear solution A. $\mathrm{Zn}(\mathrm{Ac})_{2} \cdot 2 \mathrm{H}_{2} \mathrm{O}(1.65132 \mathrm{~g})$ was dissolved in $120 \mathrm{~mL}$ DMF to form another clear solution $\mathrm{B}$. Later, solution B was added to solution A slowly under stirring, after that the mixture solution was sealed and vigorously stirred for about $1 \mathrm{~h}$ at room temperature. After centrifugation, the product was washed with ethanol for three times and dried at $60{ }^{\circ} \mathrm{C}$, and finally the JUC-160 was then obtained.

\subsection{Preparation of cobalt doped JUC-160}

De novo synthesis: In a typical synthesis, benzimidazole $(0.29541 \mathrm{~g}), 2$-methylbenzimidazole $(0.26439 \mathrm{~g})$ and a certain amount of cobalt chloride hexahydrate $\left(\mathrm{CoCl}_{2} \cdot 6 \mathrm{H}_{2} \mathrm{O}\right)$ were dissolved in $40 \mathrm{ml}$ DMF (N,N-Dimethylformamide) and stirred at ambient condition for 20 min to form a clear solution A. $\mathrm{Zn}(\mathrm{Ac})_{2} \cdot 2 \mathrm{H}_{2} \mathrm{O}(0.49357 \mathrm{~g})$ was dissolved in $30 \mathrm{ml} \mathrm{DMF}$ to form another clear solution B. Later, solution B was added to solution A slowly under stirring, after that the mixture solution was sealed and vigorously stirred for $24 \mathrm{~h}$ at room temperature. After centrifugation, the product was washed with ethanol for three times and dried at $70{ }^{\circ} \mathrm{C}$, and finally the cobalt-doped JUC-160 (CJ) was then obtained. Due to the addition of $\mathrm{CoCl}_{2} \cdot 6 \mathrm{H}_{2} \mathrm{O}$, the resulting products are denoted as CJ-n (Table S2), where $\mathrm{n}$ refers to the quantity of $\mathrm{CoCl}_{2} .6 \mathrm{H}_{2} \mathrm{O}(0.03,0.05$ and $0.07 \mathrm{~g})$. 
The post-synthetic treatment of JUC-160 (PCJ) would be realized by adding a certain amount of JUC-160 $(0.5,0.8$ and $1.1 \mathrm{~g})$ and $\mathrm{CoCl}_{2} \cdot 6 \mathrm{H}_{2} \mathrm{O}(0.06 \mathrm{~g})$ into $40 \mathrm{ml}$ ethanol. The mixed liquor was then stirred vigorously for $24 \mathrm{~h}$ at room temperature, after centrifugation, the PCJ samples were obtained. Based on the addition of JUC-160 (Table S3), the samples are named as PCJ$\mathrm{m}(\mathrm{m}=0.5,0.8$ and 1.1$)$.

\subsection{Preparation of $\mathrm{CNC}-\mathrm{n} / \mathrm{SeS}_{2}$ and $\mathrm{PCNC}-\mathrm{m} / \mathrm{SeS}_{2}$}

In order to obtain the cobalt/nitrogen co-doped carbon materials, the as-synthesized CJ-n and PCJ-m were further carbonized by annealing at $900{ }^{\circ} \mathrm{C}$ for $5 \mathrm{~h}$ under $\mathrm{Ar}$ atmosphere. The inactive cobalt particles were removed by washing with hydrochloric acid (10\%). After being washed with deionized water to wipe off residual acid, the samples were centrifugated and dried at $70{ }^{\circ} \mathrm{C}$ for $12 \mathrm{~h}$, and then the carbon materials co-doped with cobalt and nitrogen were obtained. The CNC-n/ $\mathrm{SeS}_{2}$ and $\mathrm{PCNC}-\mathrm{m} / \mathrm{SeS}_{2}$ were prepared using the mixture of carbon materials (CNC-n and PCNC-m) and SeS 2 . For the samples of CNC-n/SeS 2 and PCNC-m/SeS 2 , the weight ratio of carbon and $\mathrm{SeS}_{2}$ is 27:73 and 3:7, respectively. After grinding in an agate mortar, the mixture was heated at $160{ }^{\circ} \mathrm{C}$ for $12 \mathrm{~h}$.

\subsection{Preparation of $\mathrm{CNC}-0.05 / \mathrm{Li}_{2} \mathrm{~S}_{6}$ for adsorptivity measurements}

In order to test the chemisorption of the electrode material on $\mathrm{Li}_{2} \mathrm{~S}_{6}$, a solution of $\mathrm{Li}_{2} \mathrm{~S}_{6}$ was prepared according to previous report [29], sulfur powder and lithium sulfide $\left(\mathrm{Li}_{2} \mathrm{~S}\right)$ with a molar ratio of 5:1 were added into commercial electrolyte that was composed of $0.1 \mathrm{M}$ $\mathrm{LiNO}_{3}$ and $1 \mathrm{M}$ bis(tri-fluoromethane) sulphonimide lithium salt (LiTFSI) in a mixture of 1,3dioxolane and 1,2-dimethoxyethane (1:1 by volume), and then finally stirred at $60{ }^{\circ} \mathrm{C}$ for $24 \mathrm{~h}$. The CNC-0.05(20 mg) was added into the dark brown solution $\left(\mathrm{Li}_{2} \mathrm{~S}_{6}, 10 \mathrm{ml}\right)$, after shelving for 30min, the sample was removed from the glove box, and it was then centrifuged and vacuum dried under $70^{\circ} \mathrm{C}$.

\subsection{Characterizations}

The scanning electron microscopy (SEM) and the energy dispersive $\mathrm{x}$-ray spectroscopy (EDS) images were collected using a field emission electron microscope (Japan, SU-70). The lowand high-magnification TEM images of the CNC-n were completed in Japan Electron Optics Laboratory (JEOL) with a JEM-F200 transmission electron microscope. The Powder X-ray diffraction (XRD) patterns were recorded in a focused optical path mode of Rigaku SmartLab $\mathrm{X}$-ray Diffractometer, and the data were collected from $2 \theta=15^{\circ}-80^{\circ}$ for CNC-n and PCNC$\mathrm{m}$, while $2 \theta=5^{\circ}-40^{\circ}$ for the precursors of CJ-n and PCJ-m. The thermo-gravimetric analysis (TGA) curves were measured using Discovery TGA 55 thermal gravimetric analyzer (USA), and the test was executed under $\mathrm{N}_{2}$ atmosphere at the temperature range of $25-800{ }^{\circ} \mathrm{C}$. The specific surface area of CNC-n and PCNC-m was tested using an ASAP 2020 plus HD88 system (Micromeritics company, USA), and the pore size distribution was calculated using the density functional theory (DFT) module in this system. The confocal Raman spectra were acquired using a RENIDHAW Raman Microscope (UK, Renishaw company). The X-ray photoelectron spectroscopy (XPS) data was obtained using a ULVAC-PHI X-ray photoelectron spectroscopy (Japan, PHI-5000 Versaprobe II (VP-II)). Fourier Transform Infrared Spectrometer (FT-IR) and Diffuse-reflectance UV-Visible (DR-UV-Vis) spectra were recorded on a Nicolet FTIR 6700 (Thermo Nicolet) and PerkinElmer Lambda 950 spectrophotometer equipped, respectively. The element analysis data was obtained by using an Elementar Vario EL CUBE (Germany). 


\subsection{Cell fabrication and measurements}

All the Li-SeS 2 batteries were assembled into CR2032 coin-type cells in a Vigor Glove-box $\left(\mathrm{O}_{2}<0.1 \mathrm{ppm} ; \mathrm{H}_{2} \mathrm{O}<0.1 \mathrm{ppm} ; \mathrm{Ar}\right.$ atmosphere $)$. The electrolyte was the mixture of 1,3dioxolane (DOL) and 1,2-dimethoxyethane (DME) (volume ratio =1:1) with $1 \mathrm{M} \mathrm{LiTFSI}$ and $0.1 \mathrm{M} \mathrm{LiNO}_{3}$ added. The Celgard 2400 was served as the separator and $\mathrm{Li}$ foil $(99.9 \%$, $15.6^{*} 0.46 \mathrm{~mm}$ ) was employed as counter electrode. The working electrode was prepared by mixing $80 \%$ of $\left(\mathrm{CNC}-\mathrm{n} / \mathrm{SeS}_{2}\right.$ or PCNC-m/SeS $), 10 \%$ of Super-P and $10 \%$ of sodium alginate (Macklin, biochemical grade) in deionized water solvent. The obtained slurry was blade casted onto an aluminium foil, and then dried at $70{ }^{\circ} \mathrm{C}$ for $1000 \mathrm{~min}$ under vacuum $(-0.1 \mathrm{Mpa})$. The areal loading of active substrate was about $1.5 \mathrm{mg} \mathrm{cm}^{-2}$. Cyclic Voltammetry (CV) and impedance spectroscopy (EIS) measurements were carried out using Shanghai Chenhua CHI660D electrochemical workstation at $25{ }^{\circ} \mathrm{C}$. The EIS was recorded in a frequency range of $10^{-2}-10^{5} \mathrm{~Hz}$, and $\mathrm{CV}$ were executed in a potential range of $1.6-2.8 \mathrm{~V}$ at a scan rate of $0.1 \mathrm{mV} \mathrm{s}^{-1}$. The constant current charge-discharge and the C-rate $\left(1 \mathrm{C}=1123 \mathrm{~mA} \mathrm{~h} \mathrm{~g}^{-1}\right)$ test of the $\mathrm{Li}_{-} \mathrm{SeS}_{2}$ batteries were carried out using a Neware battery testing system (Shenzhen, China), and the used voltage window of the Li-SeS 2 battery was 1.6-2.8 V.

\section{Results and discussion}

The morphologies of the cobalt doped JUC-160 with different cobalt-doping method and cobalt-doping amounts were observed using FESEM. It can be seen from Fig. 1 that the doping amount of $\mathrm{CoCl}_{2} \cdot 6 \mathrm{H}_{2} \mathrm{O}$ has a significant influence on the crystal structure of CJ-n. With the increase of cobalt doping, the CJ-n gradually evolved from the flaky-like of CJ-0.03 to the flower-like of CJ-0.07, and the similar evolution of crystal morphology has also been observed in previous reports, such as Zn-doped Ni-MOF [24] and Fe-doped Co-MOF [21]. In a sharp contrast, with the increase of cobalt doping, the change of morphology is not obvious for PCJ$\mathrm{m}$. This phenomenon suggests that the doping method also has a significant impact on the morphology of cobalt-doped JUC-160. 

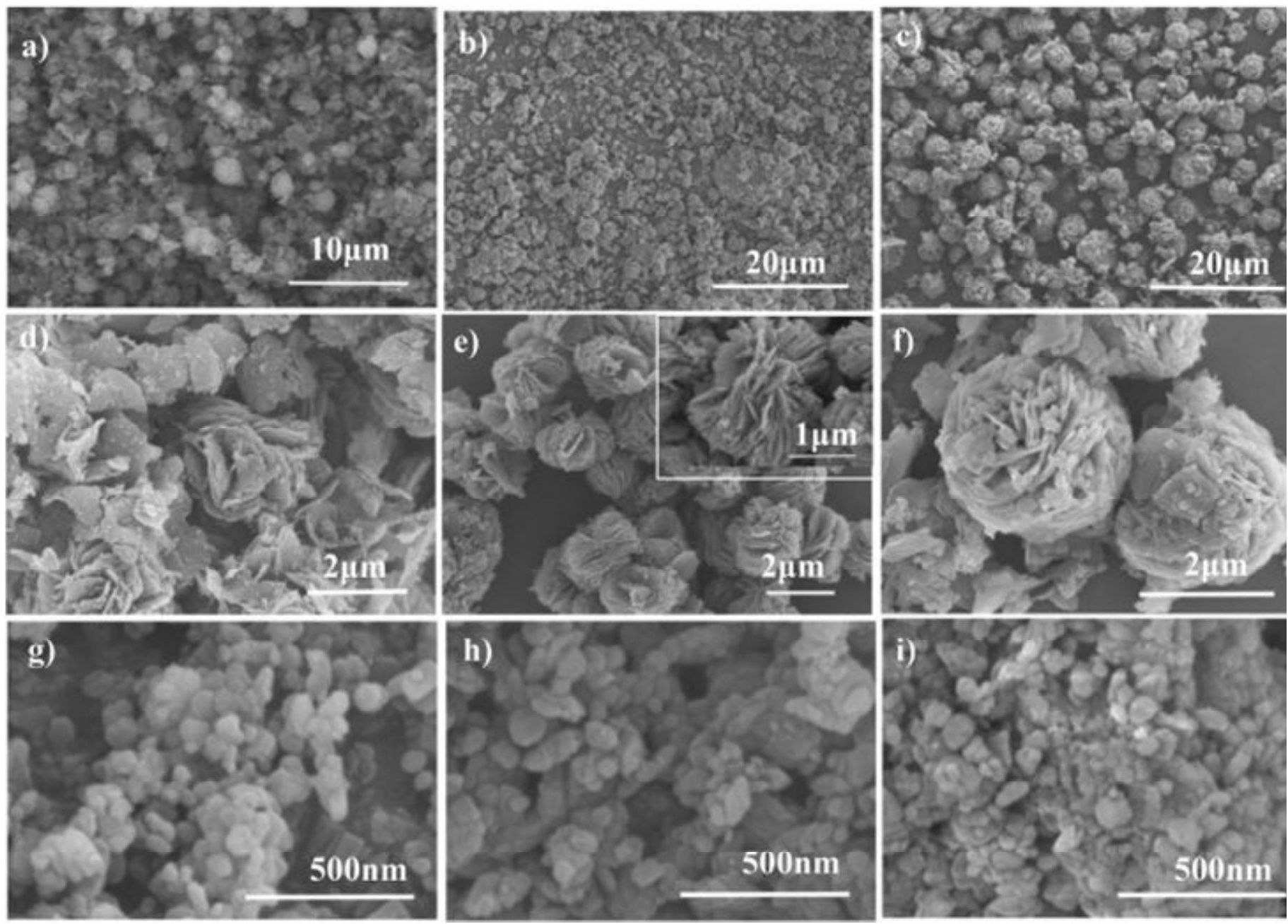

1. Download : Download high-res image (1MB)

2. Download : Download full-size image

Fig. 1. SEM image of (a,d) CJ-0.03, (b,e) CJ-0.05,(c,f) CJ-0.07, (g) PCJ-1.1,(h) PCJ-

0.8 and (i) PCJ-0.5.

To further characterize the phase composition of CJ-n and PCJ-m, powder X-ray diffraction (XRD) analysis was carried out. As seen in Fig. 2(a), the XRD patterns of CJ-n are corresponding to the characteristic peaks of JUC-160 [28]. However, an additional peak was observed at $2 \theta=10^{\circ}$, which is not corresponding to the characteristic peaks of $\mathrm{CoCl}_{2} \cdot 6 \mathrm{H}_{2} \mathrm{O}$ or organic ligands (Fig. S2). This phenomenon may result from the simultaneous competitive complexation process of $\mathrm{Co}^{2+}$ and $\mathrm{Zn}^{2+}$ ions in the synthesis process [30]. The larger $\mathrm{Zn}^{2+}$ ions $(0.074 \mathrm{~nm})$ partly replaced the smaller $\mathrm{Co}^{2+}$ ions $(0.07 \mathrm{~nm})[21,24,31,32]$, which breaks the balance of the original crystal structure and causes the formation of crystals with defective structures. Previous reports have confirmed that the crystals of MOF-5 and MOF-74 also exhibited this phenomenon [21,23,24,31,33,34], which results in some peaks being added or removed. While the post-synthetic treatment method does not change the characteristic peak of JUC-160, and the XRD characteristic peaks of PCJ-m agree well with the calculated XRD patterns of JUC-160 (Fig. 2(b)) and attributed to the strong adsorption of JUC-160 [35]. The periodic diffraction conditions of $\mathrm{CoCl}_{2} \cdot 6 \mathrm{H}_{2} \mathrm{O}$ were destroyed, which is leading to the amorphous state of $\mathrm{CoCl}_{2} \cdot 6 \mathrm{H}_{2} \mathrm{O}$ (Fig. 2(b)) [[36], [37], [38]]. 

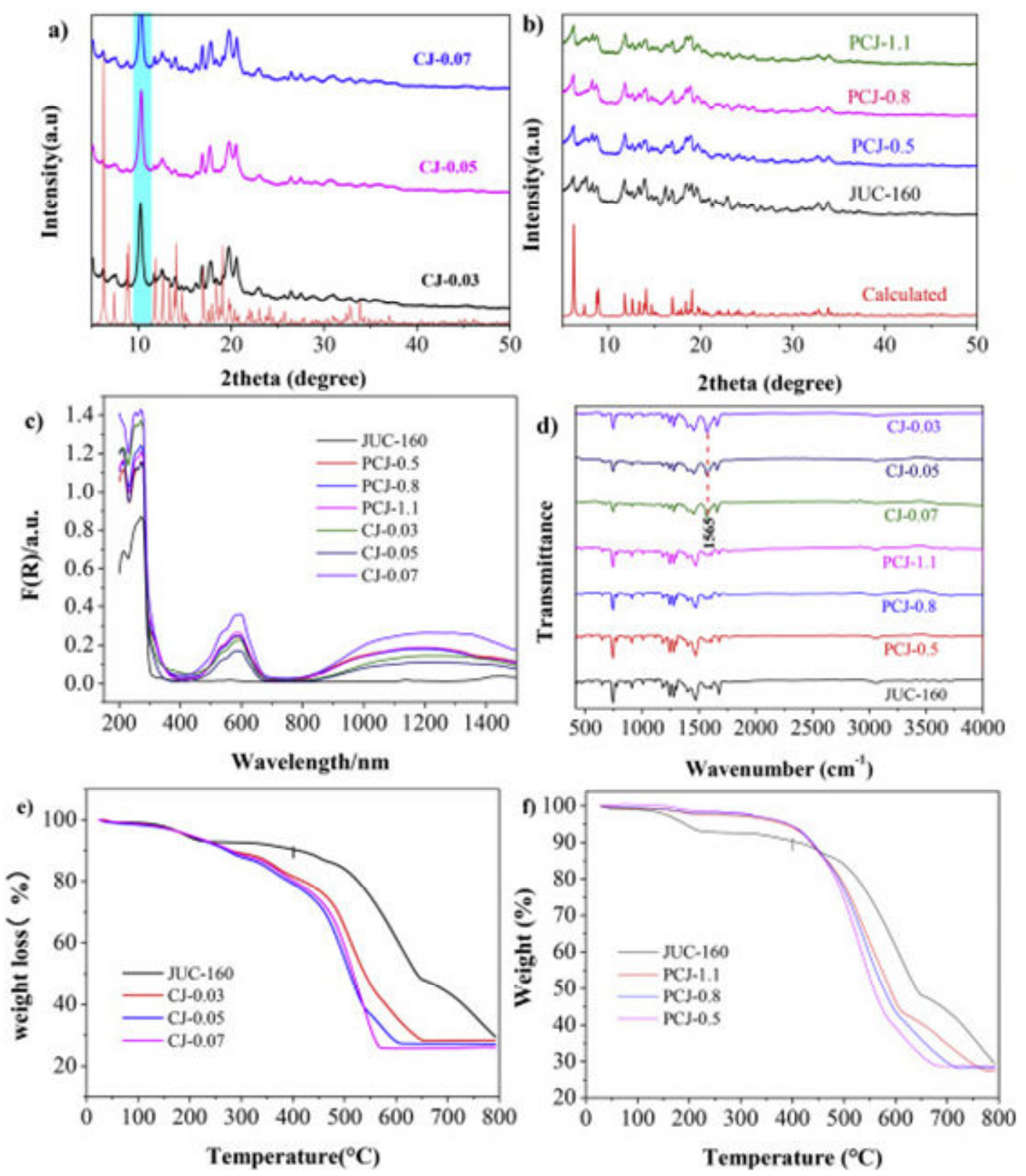

Fig. 2. (a,b) X-ray diffraction (XRD) patterns of calculated the JUC-160, CJ-n and PCJm; (c) DR-UV-Vis spectra, (d) FTIR spectra and (e,f) TGA curves of CJ-n and PCJ-m.

The DR-UV-Vis spectra of the cobalt-containing samples and JUC-160 are shown in Fig. 2(c). After cobalt doping, the CJ-n and PCJ-m show a characteristic peak of cobalt element around 400-700 [22], which fully proves that the cobalt element has been successfully doped. The formation of coordinated polymers in cobalt-containing samples was determined by FTIR spectroscopy (Fig. 2(d)). The CJ-n appears a new characteristic peak near $1565 \mathrm{~cm}^{-1}$, which suggests the strong interaction between the hydroxyl/carboxylic acid groups and $\mathrm{Co}^{2+}$ ions $[21,39]$. However, the characteristic spectrum of JUC-160 was not changed by post-synthetic treatment, and this phenomenon indicates that $\mathrm{CoCl}_{2} \cdot 6 \mathrm{H}_{2} \mathrm{O}$ is physically adsorbed in the pores of JUC-160, which further verifies the conclusion from the XRD.

Based on the related reports of crystal-shape engineering and above analysis, a possible formation path of 3D self-assembled nanostructures was proposed in Fig. 3. The CJ-n samples show similar XRD patterns, DR-UV-Vis spectra and FT-IR spectra (Fig. 2(a-d)), although they have significant differences in microscopic structures (Fig. 1(a-f)), which might result from 
their crystal growth conditions. The resulting crystals often exhibit complex forms ranging from two-dimensional (2D) chains to 3D hierarchical and self-similar superstructures. The assembling process of 3D nanostructures can be explained by the related theory of Orientated Attachment (OA) or the minimization of Gibbs free energy. Nanoparticles gradually re-arrange into higher dimensional nanostructures by sharing single crystal surface [[40], [41], [42]]. This process is influenced by diffusion kinetics of the particles in the solution system and van der Waals interactions $[40,43,44]$. In this case, the introduction of cobalt broke the dynamic balance of the MOF nucleation process. In order to complete the nucleation in the adjusted solvent system, the diffusion rate is fast enough to cause the particles to arrange in the form of the lowest energy, and van der Waals interactions could lead to further attraction [40,43,44]. This further led to the formation of more complex crystal structures, and similar phenomena have been observed in our previous reports on Al-based MOF $[43,44]$.

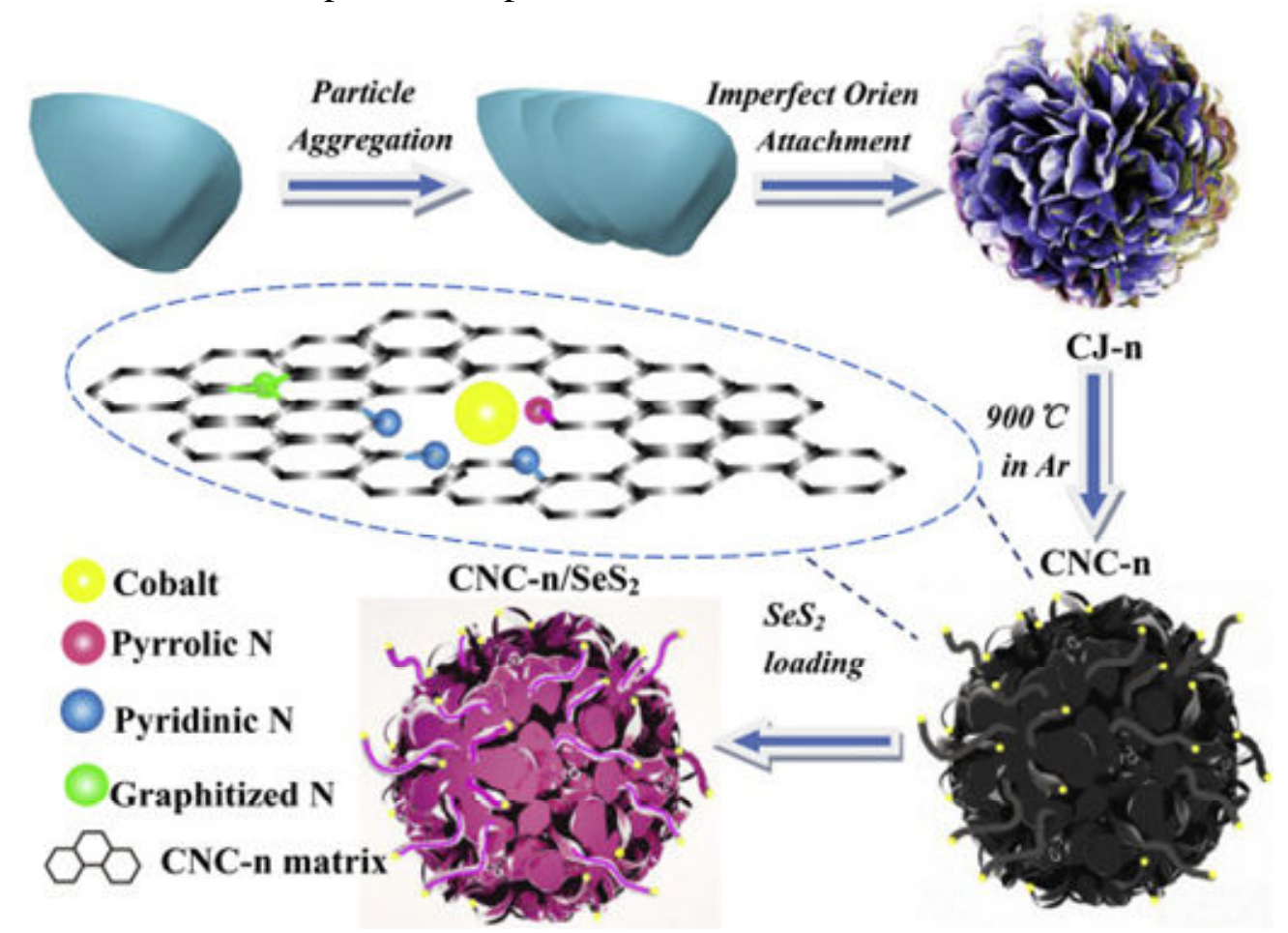

Fig. 3. Illustration of the synthesis route for the $\mathrm{CNC}-\mathrm{n} / \mathrm{SeS}_{2}$ composite.

In order to prepare the carbon materials co-doped with cobalt/nitrogen, the CJ-n and PCJ-m were further carbonized. As shown in Fig. 2(e,f), the TGA curves of CJ-n and PCJ-m demonstrates two relatively obvious weightlessness processes. The volatilization of small organic molecules in the MOF occurs in the temperature range of $25-200{ }^{\circ} \mathrm{C}$. The second stage is assigned to the removal of the guest molecules and carbonization stages. For the cobaltcontaining samples prepared by DE novo synthesis method, the weight loss cut-off temperature range was $560-650{ }^{\circ} \mathrm{C}$, while, for the post-synthetic treatment method, this temperature range is moved to $680-800{ }^{\circ} \mathrm{C}$. This phenomenon proves that the cobalt doping methods have a significant effect on the thermal stability of the samples. Additionally, with the increase of cobalt doping amount, the weight loss cut-off temperature moves to lower temperature for all the cobalt-doped simples. A similar phenomenon was also observed in Ni-doped MOF [45], which suggests that the amount of polar metal cation (such as $\mathrm{Ni}^{2+}$ and $\mathrm{Co}^{2+}$ ) doping has a significant effect on the thermal stability of the MOF materials.

The phase compositions of CNC-n and PCNC-m were identified by Powder X-ray diffraction (XRD) analysis. As shown in Fig. 4(a1), JUC-160 derived carbon materials (CNC-0) exhibit the characteristic peaks of amorphous carbon. After the cobalt doping, the main diffraction peaks of cobalt-containing samples are present at $2 \theta=26.5^{\circ}, 44.1^{\circ}, 51.5^{\circ}$, and $75.8^{\circ}$, which is 
assigned to metallic cobalt (JCPDS Card No.15-0806) and graphite (JCPDS Card No. 656212 ), respectively. This phenomenon suggests that the cobalt doping promotes the formation of graphitized carbon, which is considered to be beneficial for improving the conductivity of electrode materials [46]. Meanwhile, the high dispersion of cobalt nanoparticles in the carbon matrix can effectively improve the catalytic activity of electrode materials $[10,46]$.
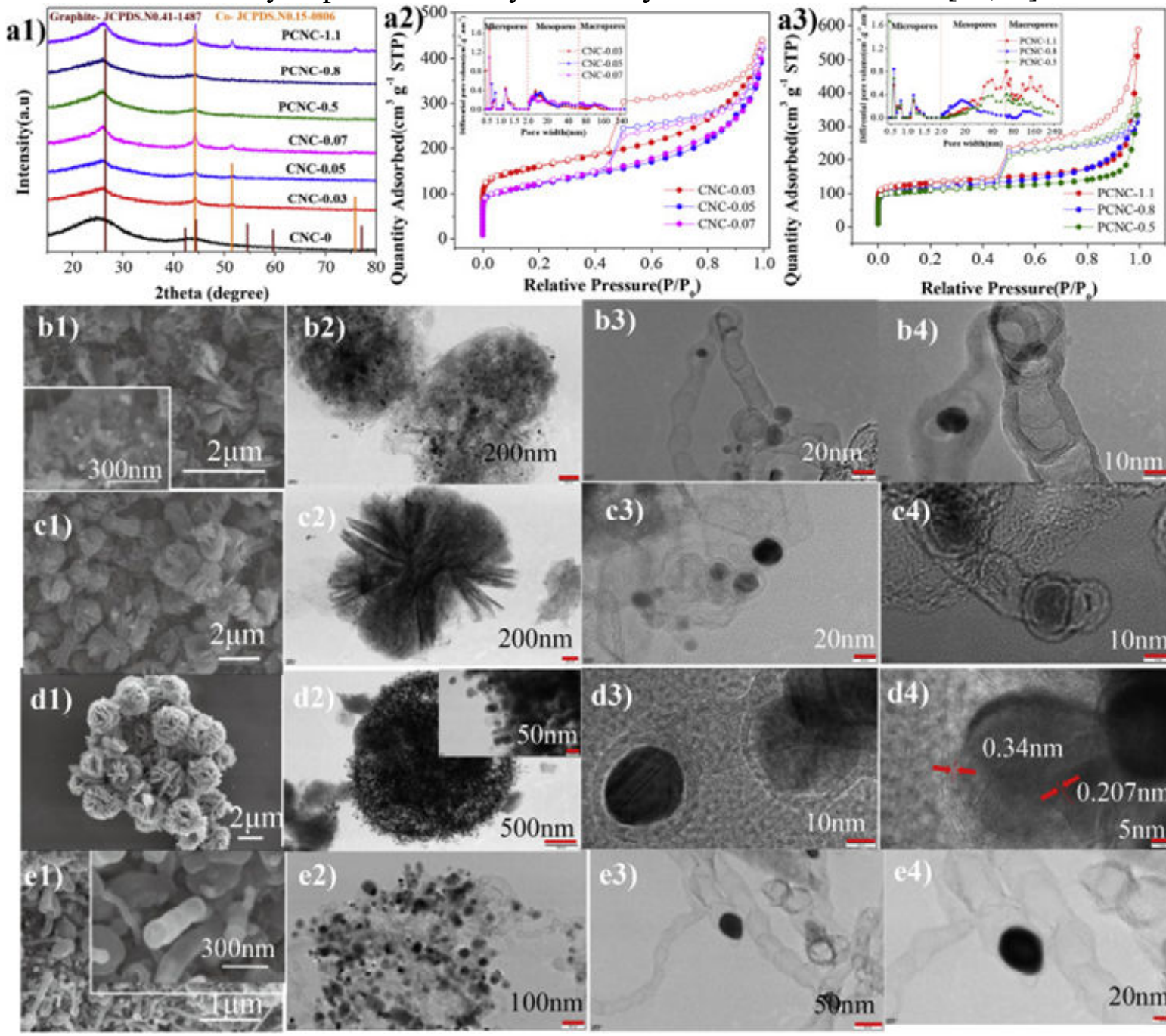

4)

Fig. 4. XRD patterns (a1), $\mathrm{N}_{2}$ adsorption-desorption isotherms and pore size distribution of CNC-n and PCNC-m using DFT analysis (a2,a3); SEM and TEM images of the CNC-0.03(b1-b4), CNC-0.05 (c1-c4), CNC-0.07 (d1-d4), and PCNC-0.8 (d1d4).

FESEM and TEM were performed to observe the morphology of CNC-n and PCNC-m. As shown in Fig. 4(b1), the structure of CNC-0.03 displays a hybrid structure of carbon nanosheets and carbon nanotubes (CNTs), which has a bamboo-like structure due to nitrogen doping [47]. It can be further seen from low-magnification TEM (Fig. 4(b2,b3)) that the nanotube was generated by cobalt catalysis. The high resolution transmission electron microscopy (HRTEM) reveals that the cobalt nanoparticles were embedded in the hollow carbon bubble structures (Fig. 4(b4)), which was resulted from the removal of partially inactive cobalt after acid treatment, and the carbon skeleton structure that was effectively reconstructed. The carbonization products of CNC-0.05 and CNC-0.07 are shown in Fig. 4(c1-c4), Fig. 4(d1-d4) and Fig. S5, and the structures of CNC-0.05 and CNC-0.07 were found to maintain the layer alignment to their parent precursors with ordered arrangements. The nanoparticles of $\mathrm{CNC}$ - 
0.05 show an open-book-like nanostructure with bamboo-like CNTs (Fig. 4(c2-c4), Figs. S5(ad)) and graphitized carbon bubble (Figs. S5(e-f)), and the CNC-0.07 has a rose-like nanostructure with CNTs (Fig. 4(d2-d4)). The representative HRTEM image of CNC-0.07 showed clear lattice fringes. The interspacing of $0.207 \mathrm{~nm}$ (Fig. 4(d4)) accounted for the (111) lattice fringes of the cobalt nano-particles, Meanwhile, the streaks of graphite at the edge of the nanosheets could be observed, and the interspacing of $0.34 \mathrm{~nm}$ (Fig. 4(d4)) accounted for the (002) lattice fringes of the graphite. The results of HRTEM further verify the conclusion from XRD that cobalt doping can improve the graphitization degree of carbon matrix. The uniform distribution of carbon, nitrogen, and cobalt was further confirmed using EDS analysis methods (Fig. S6), the cobalt content in the samples was estimated to be $4.21 \%, 4.97 \%$ and $6.41 \%$ for CNC-0.03, CNC-0.05 and CNC-0.07, respectively (Fig. S6(f1-f3)).

The morphological characteristics of PCNC-m are observed in Fig.4(e) and Fig. S7. When the amount of cobalt doping is low, cobalt sources cannot completely cover JUC-160. Therefore, only a small amount of carbon nanotubes can be observed on the surface of PCNC-1.1(Fig. S7(a)), while due to the suitable cobalt/carbon ratio of PCNC-0.8, the carbon nanotubes derived from cobalt-catalyzing can be more uniformly distributed on the surface of carbon matrix (Fig. S7(b)). Similar to CNC-n, the TEM of PCNC-0.8 reveals that the CNTs have bamboo-like structures with cobalt nanoparticle-embedded in the top (Fig. 4(e1-e4)). When the proportion of cobalt/JUC-160 was further improved, the serious agglomeration was observed in PCJ-0.5 (Fig. S7(c)), as a result, the PCJ-0.5 derived carbon nanotubes exhibit heterogeneous distribution. The cobalt content in the PCNC-m was estimated to be $2.82 \%, 3.18 \%$ and $6.14 \%$ for PCNC-1.1, PCNC-0.8 and PCNC-0.5, respectively (Fig. S8(f1-f3)). It is interesting to note that the post-synthetic treatment method does not change the crystal structure of the JUC-160 (Fig. 2(b,d)). However, the structure of their carbon materials is obviously different, which further suggests that the amount of cobalt doping also has a significant impact on the cobalt/nitrogen co-doped carbon structures.

The elemental analysis was further performed to determine the elemental composition of the CNC-n and PCNC-m. It can be found that the carbonized samples inherit the high $\mathrm{N}$ content of precursors. However, due to the breakage of $\mathrm{C}-\mathrm{N}$ bond during high temperature carbonization $\left(\geqq 850{ }^{\circ} \mathrm{C}\right)$ [45], the nitrogen content of carbonized samples was decreased significantly (Table 1 and Table 2).

Table 1. Elemental Analysis Results for different precursors.

\begin{tabular}{lllll} 
samples & $\mathbf{N}[\mathbf{w} \%]$ & $\mathbf{C}[\mathbf{w t} \%]$ & $\mathbf{H}[\mathbf{w t} \%]$ & $\mathbf{S}[\mathbf{w t} \%]$ \\
\hline JUC-160 & 17.11 & 54.43 & 4.25 & 0 \\
PCJ-0.5 & 14.32 & 49.8 & 4.445 & 0 \\
PCJ-0.8 & 16.57 & 53.34 & 4.242 & 0 \\
PCJ-1.1 & 16.86 & 52.82 & 4.166 & 0 \\
CJ-0.07 & 14.41 & 53.89 & 3.9 & 0 \\
CJ-0.05 & 14.68 & 50.29 & 4.302 & 0 \\
CJ-0.03 & 16.92 & 49.7 & 4.297 & 0
\end{tabular}


Table 2. Elemental Analysis Results for carbon materials.

$\begin{array}{lllll}\text { samples } & \mathbf{N}[\mathbf{w t} \%] & \mathbf{C}[\mathbf{w t} \%] & \mathbf{H}[\mathbf{w t} \%] & \mathbf{S}[\mathbf{w t} \%] \\ \text { CNC-0 } & 5.04 & 70.07 & 2.094 & 0 \\ \text { PCNC-0.5 } & 3.15 & 72.87 & 1.837 & 0 \\ \text { PCNC-0.8 } & 4.13 & 73.25 & 1.724 & 0 \\ \text { PCNC-1.1 } & 4.35 & 72.67 & 1.644 & 0 \\ \text { CNC-0.07 } & 2.5 & 77.99 & 1.446 & 0 \\ \text { CNC-0.05 } & 2.97 & 80.22 & 1.286 & 0 \\ \text { CNC-0.03 } & 4.05 & 74.31 & 1.465 & 0\end{array}$

The nitrogen adsorption-desorption isotherms of the CNC-n and PCNC-m are shown typical type-I/IV curves (Fig. 4(a2,a3)), which indicates that the cobalt/nitrogen co-doped carbon nanostructures have hierarchical porous structure [46], which agrees well with the results of porous distribution (Fig. 4(a2,a3)). Compared with JUC-160 derived carbon structure with abundant micropores [28], there are more mesopores $(2-50 \mathrm{~nm})$ and macropores $(>50 \mathrm{~nm})$ in the pore distribution of CNC-n and PCNC-m (Fig. 4(a2,a3) and Table .3). With the increase of cobalt content, the specific surface area of the cobalt-containing carbon materials displays a downward trend, and this phenomenon results from a significant difference in the boiling points of the cobalt and zinc $\left(\right.$ zinc $=907{ }^{\circ} \mathrm{C} \mathrm{Vs} \mathrm{cobalt}=2870{ }^{\circ} \mathrm{C}$ ). When the temperature reaches or approaches the boiling point of zinc, it will vaporize along with the argon flow, and the specific surface area of carbon materials is significantly increased during this process [28]. In sharp contrast, with the increase of cobalt doping, the remaining metal cobalt is trapped in the carbon matrix (Fig. 4).

Table 3. BET surface area, pore size, and pore volume of the CNC-n and PCNC-m.

$\begin{array}{llll}\text { CNC-n/PCNC-m } & \text { pore size }(\mathbf{n m}) & \text { pore volume }\left(\mathbf{c m}^{3} \mathbf{g}^{-1}\right) & \text { surface } \operatorname{area}\left(\mathbf{m}^{2} \mathbf{g}^{-1}\right) \\ \text { CNC-0.03 } & 4.84 & 0.64 & 530 \\ \text { CNC-0.05 } & 5.90 & 0.61 & 416 \\ \text { CNC-0.07 } & 5.92 & 0.60 & 408 \\ \text { PCNC-0.5 } & 5.88 & 0.51 & 350 \\ \text { PCNC-0.8 } & 5.19 & 0.48 & 372 \\ \text { PCNC-1.1 } & 7.45 & 0.78 & 423\end{array}$

Given self-assembled porous nanostructure and abundant polar metal/heteroatom co-doping, as a proof concept application, the cathode materials of the $\mathrm{Li}-\mathrm{SeS} \mathrm{S}_{2}$ batteries were prepared (Fig. 3). As shown in Fig. 5(a-c), the composite materials of the $\mathrm{CNC}-\mathrm{n} / \mathrm{SeS}_{2}$ remain the original structure of carbon matrix without agglomeration. Meanwhile, the elemental mapping of the $\mathrm{CNC}-\mathrm{n} / \mathrm{SeS}_{2}$ (Figs. S9-S11) indicates the homogeneous distribution of cobalt, nitrogen, carbon, selenium and sulfur in these samples. In contrast, it was found that with the increase of cobalt doping, the samples of PCNC-m/SeS 2 gradually display an obvious agglomeration (Fig. 
5(d-f)), and the residual $\mathrm{SeS}_{2}$ can be observed on the surface of the PCNC-0.5/SeS , and this phenomenon is mainly due to the fact that the specific surface area of PCNC-m decreases significantly with the increase of cobalt doping amount (Fig. 4(a3), Table .3). The specific surface area of the PCNC-0.5 was only $350 \mathrm{~m}^{2} \mathrm{~g}^{-1}$.
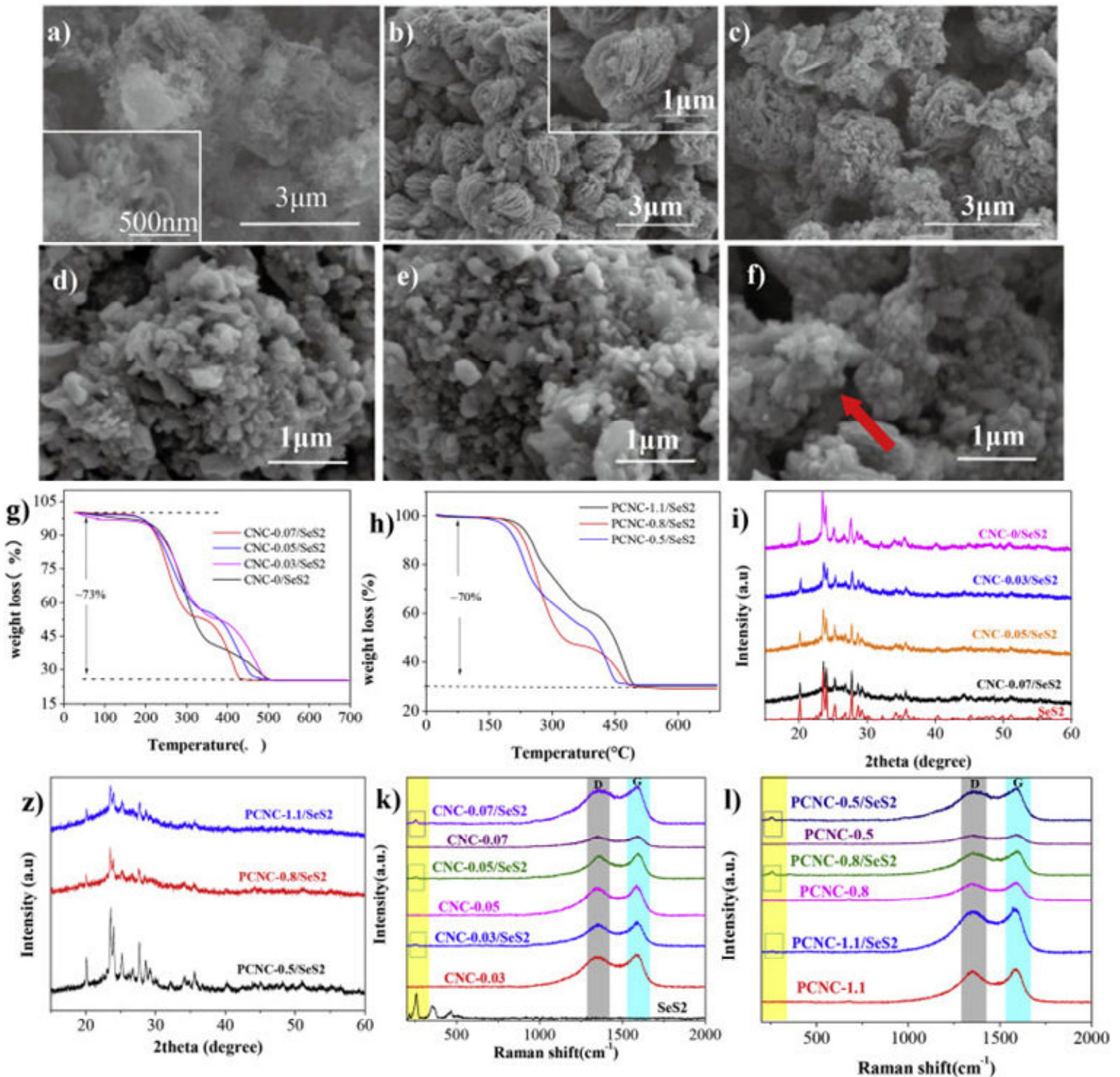

Fig. 5. SEM image of (a) CNC-0.03/SeS 2 , (b) CNC-0.05/SeS $\mathrm{C}_{2}$, (c) CNC-0.07/SeS ${ }_{2}$, (d) PCNC-1.1/SeS 2 , (e) PCNC-0.8/SeS 2 , (f) PCNC-0.5/SeS 2 ; (g,h) TGA curves, (i,z) XRD patterns of CNC-n/SeS $\mathrm{S}_{2}$ and PCNC-m/SeS $\mathrm{S}_{2}$ composites; (k,l) Raman spectra of CNCn,PCNC-m, CNC-n/SeS 2 and PCNC-m/SeS 2 .

The $\mathrm{SeS}_{2}$ content in the CNC-n/SeS${ }_{2}$ and PCNC-m/SeS $\mathrm{S}_{2}$ composite materials was about $73 \%$ and $70 \%$ (Fig. 5(g,h)), respectively. Meanwhile, it can be observed that the TGA curves show two obvious weightlessness processes, which is similar to the reports of $\mathrm{He}$ et al. [10] The first stage of weightlessness ends in the temperature range of $345-360{ }^{\circ} \mathrm{C}$, which is caused by the loss of $\mathrm{SeS}_{2}$ located on the surface of carbon matrix and the large pores. Besides, due to the strong physical adsorption of $\mathrm{SeS}_{2}$ in the micro-pore structure, the second stage of 
weightlessness process is observed, and this kind of TGA curve further verifies that the carbon materials prepared in this experiment have hierarchical porous structure.

A powder X-ray diffraction (XRD) analysis was carried out to characterize the distribution of $\mathrm{SeS}_{2}$ in these composites (Fig. 5(i z)). Due to the high content of $\mathrm{SeS}_{2}$, the diffraction peaks of $\mathrm{SeS}_{2}$ are visible in these composites, implying that the crystalline state of $\mathrm{SeS}_{2}$ was physically trapped and crystallized within the pore nanostructure, which will further lead to two-stage weightlessness on the TGA curve.

After loaded with $\mathrm{SeS}_{2}$, two peaks of the carbonaceous materials $\left(1350\right.$ and $\left.1592 \mathrm{~cm}^{-1}\right)$ in Raman spectra were observed in all the composite materials of CNC-n/SeS $\mathrm{S}_{2}$ and PCNC$\mathrm{m} / \mathrm{SeS}_{2}$ (Fig. 5(k,l), Fig. S12). Besides, due to the transformation of the ring-structured $\mathrm{SeS}_{2}$ molecules into chain-like $\mathrm{Se}_{\mathrm{x}} \mathrm{S}_{\mathrm{y}}$ molecules, three obvious characteristic peaks of bare $\mathrm{SeS}_{2}$ were transformed into one characteristic peak $\left(228 \mathrm{~cm}^{-1}\right)$ in all the composites [8], and this phenomenon suggests that the $\mathrm{SeS}_{2}$ was successfully impregnated into the carbon matrix. To examine the potential of $\mathrm{CNC}-\mathrm{n} / \mathrm{SeS} \mathrm{S}_{2}$ and $\mathrm{PCNC}-\mathrm{m} / \mathrm{SeS}_{2}$ as cathode materials, the rate performance of various cathodes was measured (Fig. 6(a)). Compared with $\mathrm{CNC}-0 / \mathrm{SeS}_{2}$, the cobalt-doped cathode with De novo synthesis method displays a better rate performance. Even if the current was increased to $10 \mathrm{C}$, the reversible specific capacity of CNC-0.03/ $\mathrm{SeS}_{2}, \mathrm{CNC}$ $0.05 / \mathrm{SeS}_{2}$ and $\mathrm{CNC}-0.07 / \mathrm{SeS}_{2}$ can maintain at $203.9,158.9$ and $234.0 \mathrm{~mA} \mathrm{~h} \mathrm{~g}^{-1}$, respectively. While, for the samples prepared by post-synthetic treatment method, only the PCNC$0.8 / \mathrm{SeS}_{2}$ with moderate cobalt doping displays a better electrochemical performance than $\mathrm{CNC}-0 / \mathrm{SeS}_{2}$. In contrast, the other two samples show a decrease in the rate performance. The difference of electrochemical performance is mainly due to the influence of cobalt doping on the physical/chemical properties of cathode materials, and a similar phenomenon has been observed in our previous reports on cobalt-based MOF [44]. The physical and chemical properties of the electrode can be effectively tailored by controlling the synthesis conditions. 

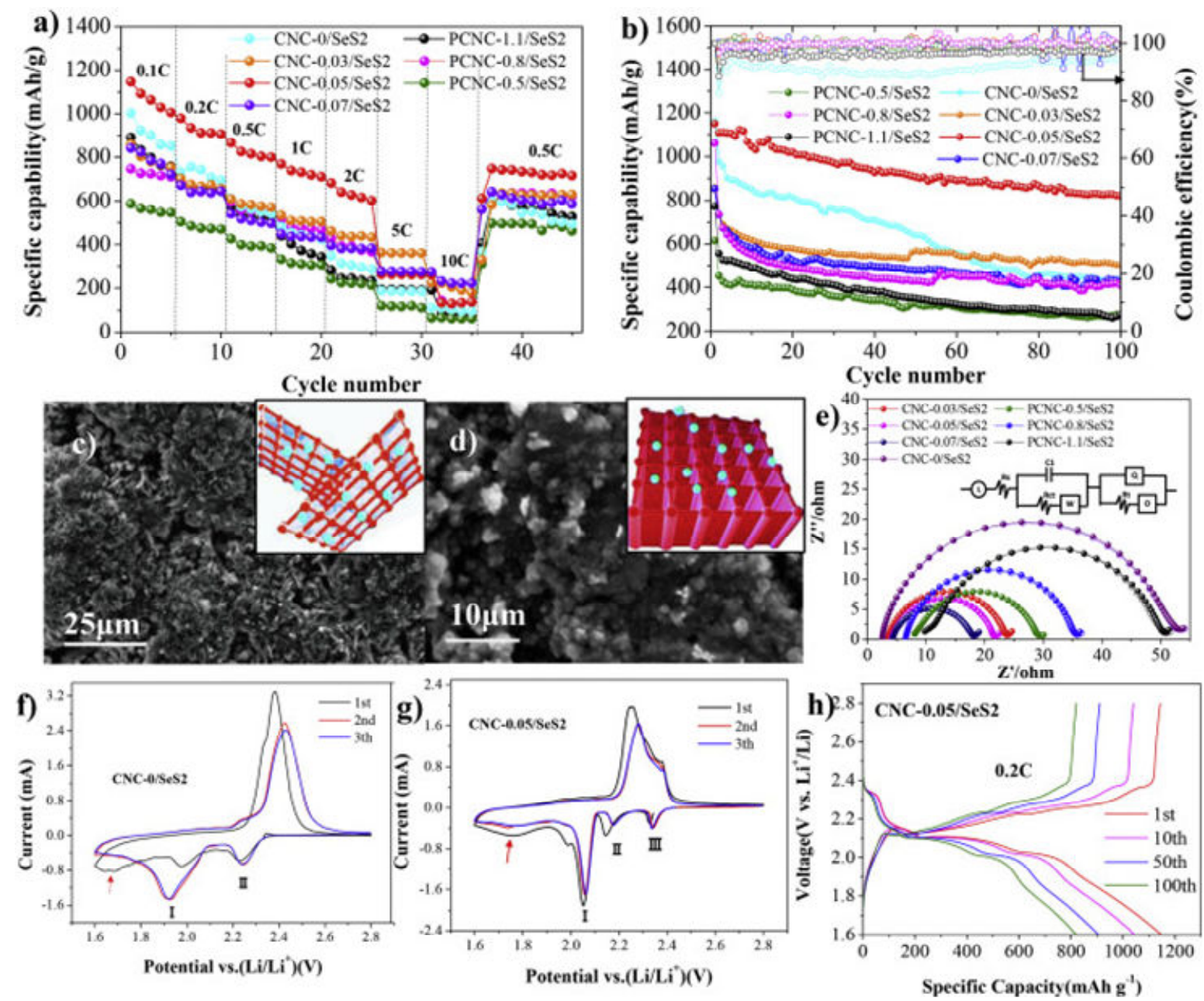

Fig. 6. (a) Rate performance of the $\mathrm{CNC}-\mathrm{n} / \mathrm{SeS}_{2}$ and $\mathrm{PCNC}-\mathrm{m} / \mathrm{SeS}_{2}$; (b) Cycle performance of the $\mathrm{CNC}-\mathrm{n} / \mathrm{SeS} \mathrm{S}_{2}$ and $\mathrm{PCNC}-\mathrm{m} / \mathrm{SeS}_{2}$ at $0.2 \mathrm{C}$; the cycled cathode of $\mathrm{c}$ ) $\mathrm{CNC}-0.05 / \mathrm{SeS}_{2}$ and d) $\mathrm{CNC}-0 / \mathrm{SeS}_{2}$. The blue ball represents $\mathrm{Li}$-ion, and the red network represents cathode materials; (e) the equivalent circuit for $\mathrm{Li}-\mathrm{SeS}_{2}$ batteries, hollow circle denotes experimental data while the solid circle represents the fitted data; $(\mathrm{e}, \mathrm{f}) \mathrm{CV}$ curve of the $\mathrm{CNC}-0 / \mathrm{SeS}_{2}$ and $\mathrm{CNC}-0.05$; (h) the voltage profiles of the CNC$0.05 / \mathrm{SeS}_{2}$ at $0.2 \mathrm{C}$ for the $1 \mathrm{st}, 10 \mathrm{th}, 50$ th and 100 th cycles, respectively. (For interpretation of the references to color in this figure legend, the reader is referred to the Web version of this article.)

In order to further test the cycling stability of cobalt-containing electrode materials, the cycle performance of the $\mathrm{CNC}-\mathrm{n} / \mathrm{SeS}_{2}$ and $\mathrm{PCNC}-\mathrm{m} / \mathrm{SeS}_{2}$ was evaluated. As shown in Fig. 6(b), CNC-0.05/SeS 2 displays an extraordinary cycle performance with $820.87 \mathrm{~mA} \mathrm{~h} \mathrm{~g}^{-1}$ reversible capacity after 100 cycles at $0.2 \mathrm{C}$, while the $\mathrm{CNC}-0.07 / \mathrm{SeS}_{2}$ displays only $426.21 \mathrm{~mA} \mathrm{~h} \mathrm{~g}^{-1}$. The long cycle performance of $\mathrm{CNC}-0.05 / \mathrm{SeS}_{2}$ was further evaluated at $0.5 \mathrm{C}$ for 250 cycles. As shown in Fig. S13, the CNC-0.05/SeS 2 displays a reversible capacity of $760.93 \mathrm{~mA} \mathrm{~h} \mathrm{~g}^{-1}$ with reversible charge-discharge efficiency close to $100 \%$. The significant difference might be due to the influence of cobalt doping on electrode structure. It can be seen from Fig. 4 that a large number of cobalt particles are wrapped in CNC-0.07. However, the CNC-0.03 and CNC-0.05 have larger space reserved between interlayers, which are more conducive to the exposure of active catalytic sites [48]. When PCNC-m/SeS${ }_{2}$ is used as cathode materials, similar results are 
observed, and PCNC-0.8/SeS 2 with moderate cobalt doping shows better cycle performance

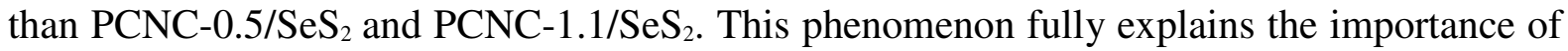
moderate cobalt doping, which can effectively balance the physical adsorption of porous carbon and the chemical anchoring of cobalt for polysulfide/polyselenium [10,49].

The morphology of the CNC-0.05/ $\mathrm{SeS}_{2}$ cathode materials is further characterized after 100 cycles at $0.2 \mathrm{C}$, except for a slight volume expansion, the structure of the electrode materials remains unchanged, and no obvious cracking or pulverization can be observed (Fig. S14). In addition, it was observed that SA has no considerable effect on the phase composition of $\mathrm{SeS}_{2}$, after 100 cycles at $0.2 \mathrm{C}$, the XRD signals of the electrode have no obvious change (Fig. S15), which is consistent with previous reports $[12,50]$ and this further confirmed that the CNC$0.05 / \mathrm{SeS}_{2}$ electrode has high electrochemical stability and the highly reversible conversion of $\mathrm{SeS}_{2}$. Moreover, after cycling, the surface structural features of the cycled cathode were further observed. As shown in Fig. 6(c,d), the $\mathrm{CNC}-0.05 / \mathrm{SeS}_{2}$ displays a scaly-like surface structure with abundant reserved space between the lamellae. However, the $\mathrm{CNC}-0 / \mathrm{SeS}_{2}$ cathode shows a dense packing morphology, which is considered to be extremely detrimental to Li-ion diffusion [51]. Meanwhile, the microporous structure of CNC-0 leads to the insufficient utilization of polar adsorption sites. The above comparison further highlights the structural advantages of the $\mathrm{CNC}-0.05 / \mathrm{SeS}_{2}$ cathodes in improving Li storage performance. Besides, the uniform distribution of carbon, cobalt, nitrogen, selenium, and sulfur elements was further confirmed by EDS tests after the cycling tests of the $\mathrm{CNC}-0.05 / \mathrm{SeS}_{2}$ cathodes. (Fig. S16).

Electrochemical impedance spectra (EIS) analysis was carried out confirming the effect of cobalt doping on electrode conductivity (Fig. 6(e)), and the semicircle diameter in intermediate frequency region can reflect the resistance of cathode materials [47]. It further proves that the cobalt doping improves the conductivity of cathode materials, with the increase of cobalt doping amount, the conductivity of the cathode increases obviously, and this phenomenon might be attributed to the improvement of carbon matrix conductivity by cobalt catalytic graphitization.

Motivated by the excellent cycling and rate performance of $\mathrm{CNC}-0.05 / \mathrm{SeS}_{2}$, the cyclic voltammetry $(\mathrm{CV})$ curves of $\mathrm{CNC}-0.05 / \mathrm{SeS}_{2}$ and $\mathrm{CNC}-0 / \mathrm{SeS}_{2}$ were tested at a sweeping rate of $0.1 \mathrm{mV} \mathrm{s}^{-1}$. The cobalt catalysis effect and the kinetic mechanism of electrochemical redox reaction were further revealed. It can be seen from Fig. $6(f, g)$ that only one oxidation peak was observed, which results from the transformation of $\mathrm{Li}_{2} \mathrm{~S} / \mathrm{Li}_{2} \mathrm{Se}$ to polysulfides/polyselenides [7]. After the first cycle, the oxidation peak of these two samples was moving to a higher voltage, and this phenomenon is thought to be caused by the lithiation process [7], which leads to the volume expansion of cathode materials. Additionally, the reduction peak indicated by the red arrow will disappear after the first cycle, and this phenomenon is considered to be the conversion of $\mathrm{Se}_{\mathrm{x}} \mathrm{S}_{\mathrm{y}}$ to polysulfides/polyselenides [52]. It was also found that $\mathrm{CNC}$ $0.05 / \mathrm{SeS}_{2}$ has three reduction peaks, while $\mathrm{CNC}-0 / \mathrm{SeS}_{2}$ displays only two reduction peaks, which was resulted from the dissolution of high-order polysulfides/polyselenides into the electrolyte [4,7]. It suggests that the cobalt doping of $\mathrm{CNC}-0.05 / \mathrm{SeS}_{2}$ can effectively inhibit the dissolution of high-order polysulfides/polyselenides into the electrolyte. Compared with $\mathrm{CNC}-0 / \mathrm{SeS}_{2}$, the onset evoked reduction peaks of CNC-0.05/SeS $\mathrm{S}_{2}$ move towards higher voltage $(2.06 \mathrm{~V})$ and the oxidation peak moves toward a smaller voltage $(2.28 \mathrm{~V})$, and this promotes the redox potential difference of CNC-0.05/SeS 2 is being significantly lower than CNC-0/SeS and this phenomenon suggests that the presence of $\mathrm{CNC}-0.05 / \mathrm{SeS}_{2}$ can effectively reduce the electrochemical polarization [12].

Fig. 6(h) presents the discharge-charge voltage curve of the $\mathrm{CNC}-0.05 / \mathrm{SeS}_{2}$ at a rate of $0.2 \mathrm{C}$, the first discharge capacity of the $\mathrm{CNC}-0.05 / \mathrm{SeS}_{2}$ was $1149.55 \mathrm{~mA} \mathrm{~h} \mathrm{~g}^{-1}$, which is slightly higher than that of the theoretical value of $\mathrm{Li}_{-} \mathrm{SeS}_{2}$ battery $\left(1123 \mathrm{~mA} \mathrm{~h} \mathrm{~g}^{-1}\right)$, and this phenomenon can be attributed to the reduction of $\mathrm{LiNO}_{3}$ [53], which is beneficial for the 
formation of SEI film on the surface of CNC-0.05/SeS ${ }_{2}$ [54]. Four plateaus at 2.34, 2.16, 2.10 and $2.03 \mathrm{~V}$ were observed in the first discharge curves, and this value is very close to the results of CV test and previous reports [[10], [11], [12], [13]], in which the upper branch (2.34 and $2.16 \mathrm{~V}$ ) indicates the formation of soluble long-chain polysulfides and polyselenides $\left(\mathrm{Li}_{2} \mathrm{Se}_{\mathrm{n}}\right.$ and $\left.\mathrm{Li}_{2} \mathrm{~S}_{\mathrm{n}}(4 \leq \mathrm{n} \leq 8)\right)$ [10]. The lower oblique branch $(2.10$ and $2.03 \mathrm{~V})$ originates from further reduction of $\mathrm{Li}_{2} \mathrm{Se}_{\mathrm{n}}$ and $\mathrm{Li}_{2} \mathrm{~S}_{\mathrm{n}}$ to insoluble short-chain polysulfides and polyselenides $\left(\mathrm{Li}_{2} \mathrm{Se}_{2}, \mathrm{Li}_{2} \mathrm{~S}_{2}\right)[10,12]$. It is noteworthy that the movement of the charge-discharge curve is not obvious even after the 100 cycles, which indicates that the $\mathrm{CNC}-0.05 / \mathrm{SeS}_{2}$ has an excellent cycle stability and minimal polarization [12].

In order to verify the chemical interaction between the cobalt/nitrogen co-doped electrode and polysulfide, the cobalt element of $\mathrm{CNC}-0.05 / \mathrm{SeS}_{2}$ electrode was completely removed and loaded with the same $\mathrm{SeS}_{2}$ (Fig. S17). The XPS characteristic peaks of cobalt were not observed in Fig. S18, which confirmed that the cobalt element was completely removed and the samples after cobalt removal have similar element composition with $\mathrm{CNC}-0 / \mathrm{SeS}_{2}$. This is in consistent with the results of carbon matrix element analysis (Table .2). It is worth noting that the carbon matrix after $\mathrm{SeS}_{2}$ loading still contains abundant graphitized nitrogen, pyrrolic nitrogen and pyridinic nitrogen, which is believed to be beneficial to the oxidation of $\mathrm{Li}_{2} \mathrm{~S}_{\mathrm{n}}(\mathrm{n}=4-8)[10,26]$. Compared with the cycle performances of the electrode before and after cobalt removal, it can be found that the cobalt doping can significantly improve the cycle stability and chargedischarge efficiency of cathode materials (Fig. S19). Similar phenomena were also observed in previous reports $[10,26]$. This fully illustrates the importance of cobalt-nitrogen synergistic catalytic effect.

In order to more intuitively observe the chemical adsorption of CNC-0.05/SeS${ }_{2}$ on $\mathrm{S}_{\mathrm{x}}{ }^{2-}$ and $\mathrm{Se}_{\mathrm{x}}{ }^{2-}$ species, the immersion test of $\mathrm{CNC}-0.05 / \mathrm{SeS}_{2}$ electrode was carried out according to the method reported in the literature [12]. After 50 cycles at $0.2 \mathrm{C}$, the cathode plates were disassembled in an Ar-filled glovebox and soaked in the mixed solution of 1,3-dioxolane (DOL) and 1,2-dimethoxyethane (DME) (1:1 by volume) for $2 \mathrm{~h}$. It was found that the solution soaked with $\mathrm{CNC}-0.05 / \mathrm{SeS}_{2}$ electrode has a very light color, while the solution soaked with cobalt removal electrode has a slightly dark color (Fig. 7(c)), which implies the dissolution of polysulfide in organic electrolyte. To determine the limiting effect of the cathode materials on polysulfides/polyselenides, the corrosion depth of lithium selenide $\left(\mathrm{Li}_{2} \mathrm{Se}_{2}\right.$ and $\left.\mathrm{Li}_{2} \mathrm{Se}\right)$ and lithium sulfide $\left(\mathrm{Li}_{2} \mathrm{~S}_{2}\right.$ and $\left.\mathrm{Li}_{2} \mathrm{~S}\right)$ granular sediment was further observed before and after removal of cobalt. Compared with samples without cobalt doping, the lithium foil paired with the CNC-0.05/SeS 2 displays thinner depth (Fig. 7(a,b)), which is in consistent with the phenomena observed in previous reports [44,55], and this proves that the cobalt/nitrogen codoping is effective for limiting the shuttle effect of polysulfide/polyselenide. These control experiments fully prove that the optimized cathode materials need not only the physical adsorption of carbon matrix and nitrogen-doping, but also the catalytic effect of metallic cobalt. 

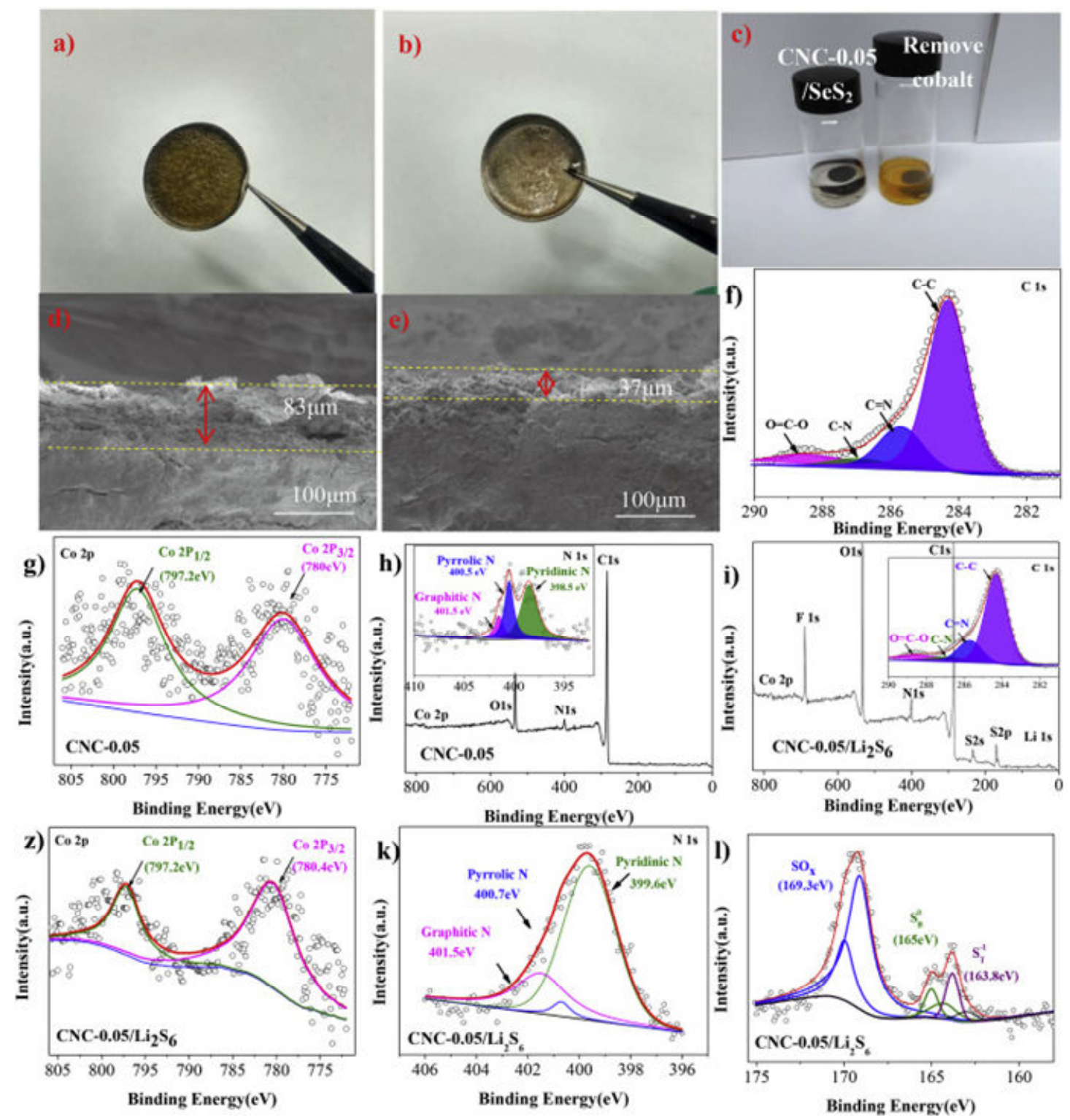

Fig. 7. (a,b) Photographs of the cycled lithium foil from cells paired with (a) remove cobalt electrodes, and (b) CNC-0.05/SeS 2 . Cross-sectional SEM morphologies of the cycled lithium foils paired with (d) remove cobalt electrodes, and (e) CNC-0.05/SeS ; (c) Visual comparison of $\mathrm{CNC}-0.05 / \mathrm{SeS}_{2}$ and remove cobalt electrodes immersed in mixed DOL/DME solutions at the fully discharged state after 50 cycles at $0.2 \mathrm{C}$, respectively; (h) XPS survey spectrum of CNC-0.05 and the corresponding highresolution XPS spectra for $\mathrm{N} 1 \mathrm{~s}$ (showing in the inset), (f) $\mathrm{C} 1 \mathrm{~s}$ and (g) Co 2p; (i) XPS survey spectrum of the $\mathrm{CNC}-0.05 / \mathrm{Li}_{2} \mathrm{~S}_{6}$ and the corresponding high-resolution XPS spectra for $\mathrm{C} 1 \mathrm{~s}$ (showing in the inset),(z)Co2p, (k) N 1s and (l) the thiosulfate and polythionate species, respectively.

To confirm the chemical anchoring effect of cobalt/nitrogen co-doped electrode on polysulfide, the XPS test was carried out (Fig. 7). After adsorption of $\mathrm{L}_{2} \mathrm{~S}_{6}$, the characteristic peaks of $\mathrm{F}$ were observed (Fig. 7(i)), which was derived from the addition of LiTFSI in the electrolyte $[29,56,57]$. In addition, the characteristic peaks of the thiosulfate and polythionate species were observed in a range of $166-175 \mathrm{eV}$ (Fig. 7(1)), which is believed to be formed by redox reaction 
between cobalt doped carbon matrix and $\mathrm{Li}_{2} \mathrm{~S}_{6}$. These results demonstrate the strong absorptivity of CNC-0.05 towards lithium polysulfides/polyselenides [10].

The characteristic peaks of pyrrolic nitrogen $(400.5 \mathrm{eV})$ and pyridinic nitrogen $(398.5 \mathrm{eV})$ both moved towards the direction of high binding energy (Fig. 7(h,k)), which may be due to the reduction of charge in the nitrogen functional group,[58] and this suggests that it is generated a chemical binding between nitrogen and lithium polymers [29]. In addition, similar phenomenon was also observed on the Co $2 \mathrm{P}_{3 / 2}$ spectrum Fig. $7(\mathrm{~g}, \mathrm{z})$, which is suggested that the chemical interactions were formed between CNC-0.05 and lithium polymers [10].

Based on the above discussion, it is fully proven that the optimized cobalt/nitrogen co-doped electrode materials have an extraordinary electrochemical performance, which may come from the following reasons: (i) the large specific surface area of $\mathrm{CNC}-0.05 / \mathrm{SeS}_{2}$ can effectively increase the load of active substances and fully expose the catalytic activity sites. (ii) the carbon skeleton network with high graphitization and nitrogen doping can effectively improve the conductivity of cathode materials. (iii) cobalt/nitrogen co-doping is beneficial to reduce the shuttle effect of polysulfur/polyselenium through synergetic catalyst effect, and the catalysis of polar metal cobalt promotes the conversion of long-chain polysulfide/polyselenium compounds to short-chain ones, and $\mathrm{N}$-doped carbon surface can facilitate the oxidation of $\mathrm{Li}_{2} \mathrm{~S}_{\mathrm{n}}$ and improve the adsorption energy of nitrogen-containing carbon matrix for polysulfide/polyselenite. Benefited from the above advantages, the cycling performance of active materials was improved. Finally, self-assembled carbon super-structures have large spaces between nanosheets and abundant hollow carbon bubbles structures, which can effectively buffer the volume expansion during charging/discharging.

\section{Conclusion}

In summary, the self-assembled carbon nanostructures derived from cobalt-doped JUC-160 exhibit a great potential for new generation $\mathrm{Li}_{-} \mathrm{SeS}_{2}$ batteries, and this carbon scaffold with novel structure and cobalt/nitrogen co-doping can effectively inhibit the diffusion of polysulfur/polyselenium through strong chemical interactions. Due to the features such as abundant pore structure and large specific surface area of carbon super-structure, the loading of active material $\left(\mathrm{SeS}_{2}\right)$ was up to $73 \%$, and the electrode damage caused by the volume expansion can be avoided. For many ZIF materials, one can try to explore its potential using this method. The findings in this work would be greatly beneficial to the development of various carbon nanostructures with polar metal/heteroatom, which can be used in energy storage, electro-catalysis, or gas separation, for example.

\section{Acknowledgements}

This work was financially supported by the Shenzhen Basic Research Program (JCYJ20190808141611189, JCYJ20170818100134570, JCYJ20160422091418366)

, Basic and Applied Research Fund of Guangdong Province (2020A1515011018), China Postdoctoral Science Foundation (2019M663054). Thanks for TEM measurement help of Instrumental Analysis Center of Shenzhen University (Xili Campus).

\section{References}

[1] N. Ding, Y.W. Lum, S.F. Chen, S.W. Chien, T.S.A. Hor, Z.L. Liu, Y. ZongJ. Mater. Chem. A, 3 (2015), pp. 1853-1857

[2]J.R. He, W.Q. Lv, Y. F. Chen, J. Xiong, K.C. Wen, C. Xu, W.L. Zhang, Y.R. Li, W. Qin, W.D. He, J. Power Sources 363(2017) 103-109.

[3]A. Abouimrane, D. Dambournet, K.W. Chapman, P.J. Chupas, W. Weng, K. Amine 
J. Am. Chem. Soc., 134 (2012), pp. 4505-4508

[4]Y.J. Cui, A. Abouimrane, J. Lu, T. Bolin, Y. Ren, W. Weng, C.J. Sun, V.A. Maroni, S.M. Heald, K. Amine,J. Am. Chem. Soc., 135 (2013), pp. 8047-8056

[5]W.C. Zhang, H.Q. Wang, N. Zhang, H.G. Liu, Z. Chen, L.J. Zhang, S.P. Guo, D. Li, J.Z. Xu, ACS Appl. Mater. Interfaces, 11 (2019), pp. 29807-29813

[6]Y. Yao, R. Xu, M.L. Chen, X.L. Cheng, S.F. Zeng, D.J. Li, X.F. Zhou, X.J. Wu, Y. Yu,A CS Nano, 13 (2019), pp. 4695-4704

[7]Q.T. Xu, H.G. Xue, S.P. Guo, Inorg. Chem. Front, 6 (2019), pp. 1326-1340

[8]Z. Li, J.T. Zhang, Y. Lu, X.W. (David) Lou, Sci. Adv, 4 (2018), p. 1687

[9]B.S. Guo, T.T. Yang, W.Y. Du, Q.R. Ma, L.Z. Zhang, S.J. Bao, X.Y. Li, Y.M. Chen, M.W . Xu, J. Mater. Chem. A, 7 (2019), pp. 12276-12282

[10]J.R. He, W.Q. Lv, Y.F. Chen, J. Xiong, K.C. Wen, C. Xu, W.L. Zhang, Y.R. Li, W. Qin, W.D. He, J. Mater. Chem. A, 6 (2018), pp. 10466-10473

[11]Z. Li, J.T. Zhang, B.Y. Guan, X.W. (David) Lou, Chem. Int. Ed, 56 (2017), pp. $16003-$ 16007

[12]T. Chen, W.H. Kong, M.T. Fan, Z.W. Zhang, L. Wang, R.P. Chen, Y. Hu, J. Ma, Z. Jin , J. Mater. Chem. A, 7 (2019), pp. 20302-20309

[13]J.T. Zhang, Z. Li, X.W. (David) Lou, Angew. Chem. Int. Ed., 56 (2017), pp. 1-7

[14] K.S. Park, Z. Ni, A.P. Cote, J.Y. Choi, R.D. Huang, F.J. Uribe-

Romo, H.K. Chae, M. O'Keeffe, O.M. Yaghi, Proc. Natl. Acad. Sci. Unit. States

Am., 103 (2006), pp. 10186-10191

[15]W.C. Lee, H.T. Chien, Y. Lo, H.C. Chiu, T.P. Wang, D.Y. Kang, ACS Appl. Mater.

Interfaces, 7 (2015), pp. 18353-18361

[16]N.Y. Cheng, L. Ren, X. Xu, Y. Du, S.X. Dou, Adv. Energy Mater, 8 (2018),

p. 1801257

[17]V.V. Butova, V.A. Polyakov, E.A. Bulanova, M.A. Soldatov, I.S. Yahia, H.Y. Zahra

n, A.F. Abd El-Rehim, H. Algarni, A.M. Aboraia, A.V. Soldatov, Microporous

Mesoporous Mater., 293 (2020), p. 109685

[18]C.H. Wang, J.H. Kim, J. Tang, M.J. Kim, H. Lim, V. Malgras, J. You, Q. Xu, J.S.

Li, Y. Yamauchi, Inside Chem., 6 (2020), pp. 19-40

[19] S. Dissegna, K. Epp, W.R. Heinz, G. Kieslich, R.A. Fischer, Adv.

Mater., 30 (2018), p. 1704501

[20] Z.L. Fang, B. Bueken, D.E. De Vos, R.A. Fischer, Angew. Chem. Int.

Ed., 54 (2015), pp. 7234-7254

[21]X.H. Zhao, B. Pattengale, D.H. Fan, Z.H. Zou, Y.Q. Zhao, J. Du, J.E. Huang, C.L. $\mathrm{Xu}$, ACS Energy Lett, 3 (2018), pp. 2520-2526

[22] J.A. Botas, G. Calleja, M. Sanchez-Sanchez, M.G. Orcajo, Langmuir, 26 (2010), pp. 5300-5303

[23] Y.X. Chen, D. Ni, X.W. Yang, C.C. Liu, J.L. Yin, K.F. Cai, Electrochim.

Acta, 278 (2018), pp. 114-123

[24] J. Yang, C. Zheng, P.X. Xiong, Y.F. Li, M.D. Wei, J. Mater. Chem. A, 2 (2014), pp. 19005-19010

[25] Z.Q. Jia, Z.H. Cui, Y.B. Tan, Z.W. Liu, X.X. Guo, Chem. Eng. J., 370 (2019), pp. 89-97

[26] Y.J. Li, J.M. Fan, M.S. Zheng, Q.F. Dong, Energy Environ. Sci., 9 (2016), pp. 1998-2004

[27]C. Liu, X.D. Huang, J. Wang, H. Song, Y.N. Yang, Y. Liu, J.S. Li, L.J. Wang, C.Z. Yu, Adv. Funct. Mater., 28 (2018), p. 1705253

[28] Y. Pan, M. Xue, M.Y. Chen, Q.R. Fang, L.K. Zhu, V. Valtchev, S.L. Qiu, Inorg.

Chem. Front, 3 (2016), pp. 1112-1118 
[29]L.L. Zhang, X. Chen, F. Wan, Z.Q. Niu, Y.J. Wang, Q. Zhang, J. Chen, ACS

Nano, 12 (2018), pp. 9578-9586

[30]Z.Y. Lu, B. Wang, Y.F. Hu, W. Liu, Y.F. Zhao, R.O. Yang, Z.P. Li, J. Luo, B. Chi, Z . Jiang, M.S. Li, S.C. Mu, S.J. Liao, J.J. Zhang, X.L. Sun, Angew. Chem., 131 (2019), pp. 2648-2652

[31] Y. Jiao, J. Pei, D.H. Chen, C.S. Yan, Y.Y. Hu, Q. Zhang, G. Chen, J. Mater. Chem. A, 5 (2017), pp. 1094-1102

[32] C.H. YoderIonic compounds: applications of chemistry to mineralogy, Ionic Compounds, Wiley, New York (2006), p. 171,

[33] J. Wang, Q. Zhong, Y.H. Xiong, D.Y. Cheng, Y.Q. Zeng, Y.F. Bu, Appl. Surf.

Sci., 483 (2019), pp. 1158-1165

[34] P.C. Banerjee, D.E. Lobo, R. Middag, W.K. Ng, M.E. Shaibani, M. Majumder, ACS Appl. Mater. Interfaces, 7 (2015), pp. 3655-3664

[35] Z. Li, P.P. Yang, S.C. Yan, Q.R. Fang, M. Xue, S.L. Qiu, ACS Appl. Mater.

Interfaces, 11 (2019), pp. 15748-15755

[36] R.A. London, M.D. Rosen, J.E. Trebes, Appl. Optic., 28 (1989), pp. 3397-3404

[37] J.C. Solem, G.C. Baldwin, Science, 218 (1982), pp. 229-235

[38] X. Zhang, Q.Q. Shi, B.X. Shen, Z.Z. Hu, X.Q. Zhang, J. Hazard

Mater., 381 (2020), p. 121003

[39]A. Vimont, J.M. Goupil, J.C. Lavalley, M. Daturi, S. Surblé, C. Serre, F. Millange, G. Férey, N. Audebrand, J. Am. Chem. Soc., 128 (2006), pp. 3218-3227

[40] D.S. Li, M.H. Nielsen, J.R.I. Lee, C. Frandsen, J.F. Banfield, J.J. De Yoreo,

Science, 336 (2012), p. 1014

[41] R.L. Penn, J.F. Banfield, Science, 281 (1998), pp. 969-971

[42] L. Bahrig, S.G. Hickey, A. Eychmüller, CrystEngComm, 16 (2014), pp. 9408-

9424

[43]W.W. Jin, B. Cheng, H.J. Li, S. Inguva, J.Z. Zou, Q. Zhang, S.Z. Zeng, G.Z. Xu, X. R. Zeng, Microporous Mesoporous Mater., 294 (2020), p. 109901

[44] W.W. Jin, H.J. Li, J.Z. Zou, S. Inguva, Q. Zhang, S.Z. Zeng, G.Z. Xu, X.R. Zeng, J. Alloys Compd., 820 (2020), p. 153084

[45] H.H. Li, W. Shi, K.N. Zhao, H. Li, Y.M. Bing, P. Cheng, Inorg. Chem., 51 (2012), pp. 9200-9207

[46] W.W. Jin, J.Z. Zou, S.Z. Zeng, S. Inguva, G.Z. Xu, X.H. Li, M. Peng, X.R. Zen, Appl. Surf. Sci., 469 (2019), pp. 404-413

[47]H. Guo, Q.C. Feng, J.X. Zhu, J.S. Xu, Q.Q. Li, S.L. Liu, K.W. Xu, C. Zhang, T.X. Li u, J. Mater. Chem. A, 7 (2019), pp. 3664-3672

[48] L. Huang, X.P. Zhang, Y.J. Han, Q.Q. Wang, Y.X. Fang, S.J. Dong, J. Mater.

Chem. A, 5 (2017), pp. 18610-18617

[49]J.R. He, Y.F. Chen, W.Q. Lv, K.C. Wen, C. Xu, W.L. Zhang, Y.R. Li, W. Qin, W.D. He, ACS Nano, 10 (2016), pp. 10981-10987

[50] C.P. Yang, S. Xin, Y.X. Yin, H. Ye, J. Zhang, Y.G. Guo, Angew. Chem. Int.

Ed., 52 (2013), pp. 8363-8367

[51]X.H. Hu, J.H. Jian, Z.S. Fang, L.F. Zhong, Z.K. Yuan, M.J. Yang, S.J. Ren, Q. Zha ng, X.D. Chen, D.S. Yu, Energy Storage Materials, 22 (2019), pp. 40-47

[52] C. Luo, Y.J. Zhu, Y. Wen, J.J. Wang, C.S. Wang, Adv. Funct. Mater., 24 (2014), pp. 4082-4089

[53]S.Z. Zeng, X.R. Zeng, W.X. Tu, Y.C. Yao, L. Yu, H.L. Wu, W.W. Jin, H.T. Huang,

J.Z. Zou, J.Mater.Chem.A, 5 (2017), pp. 23209-23220

[54] Z. Li, J.T. Zhang, H.B. Wu, X. W. (David) Lou, Adv. Energy Mater, 7 (2017), p. 1700281 
[55] J.R. He, G. Hartmann, M. Lee, G.S. Hwang, Y.F. Chen, A. Manthiram, Energy Environ. Sci., 12 (2019), pp. 344-350

[56]S.Y. Lang, Y. Shi, Y.G. Guo, R. Wen, L.J. Wan,Angew. Chem. Int. Ed., 56 (2017), pp. 14433-14437

[57]Y. Yang, X.J. Hong, C.L. Song, G.H. Li, Y.X. Zheng, D.D. Zhou, M. Zhang, Y.P. Ca i, H.X. Wang, J. Mater. Chem. A, 7 (2019), pp. 16323-16329

[58] X.L. Wang, Z.F. Hou, T. Ikeda, M. Oshima, M.A. Kakimoto, K. Terakura J. Phys. Chem. A, 117 (2013), pp. 579-589 
2020-07-03

\title{
Cobalt doped JUC-160 derived
}

\section{functional carbon superstructures with synergetic catalyst effect for Li-SeS2 batteries}

\author{
Jin, Wen-Wu
}

Elsevier

Jin W-w, Li H-J, Zou J-z, et al., (2020) Cobalt doped JUC-160 derived functional carbon superstructures with synergetic catalyst effect for Li-SeS2 batteries. Microporous and Mesoporous Materials, Volume 306, October 2020, Article number 110438

https://doi.org/10.1016/j.micromeso.2020.110438

Downloaded from Cranfield Library Services E-Repository 